\title{
Reforma del modelo educativo de la universidad nacional de agricultura (UNA) Catacamas, Olancho, Honduras
}

\author{
Reform of the educational model National Agricultural \\ University (UNA) Catacamas, Olancho, Honduras
}

\author{
Hernández Cubas, Mario Roberto; Zúniga-González, Carlos Alberto; \\ Editor Academico Dr. Angel Sol Sanchez
}

\author{
Mario Roberto Hernández Cubas \\ mariocubas87@yahoo.com \\ UNA Honduras, Honduras \\ Carlos Alberto Zúniga-González \\ czuniga@ct.unanleon.edu.ni \\ Universidad Nacional Autónoma de Nicaragua, León., \\ Nicaragua \\ Editor Academico Dr. Angel Sol Sanchez \\ Colegio de Postgraduados Mexico, México
}

Revista Iberoamericana de Bioeconomía y Cambio Climático

Universidad Nacional Autónoma de Nicaragua, León, Nicaragua ISSN-e: 2410-7980

Periodicidad: Semestral

vol. 2, núm. 1, 2016

czuniga@ct.unanleon.edu.ni

Recepción: 15 Febrero 2015

Aprobación: 20 Abril 2016

URL: http://portal.amelica.org/ameli/journal/394/3941750021/

DOI: https://doi.org/10.5377/ribcc.v2i1.5698

Autor de correspondencia: mariocubas87@yahoo.com
Resumen: El presente trabajo tiene como objetivo estudiar y analizar el proceso de reforma del modelo educativo de la Universidad Nacional de Agricultura, UNA-Honduras. En tal sentido, se realizaron entrevistas a diferentes actores tales como docentes, egresados y alumnos para que brindaran su punto de vista del modelo educativo de la UNA, así mismo se efectuaron jornadas de reflexión con expertos en el área educativa para analizar el sistema que posee la universidad en el ámbito educativo, la reforma se apegó al proyecto tunning para latino américa el cual tiene estructurada la metodología que facilita la comprensión de los planes de estudios académicos y su mutua comparación. Desde este escenario, la Universidad Nacional de Agricultura (UNA-Honduras), con los insumos obtenidos desde diversas fuentes construirá el modelo educativo que se sustenta en concepciones teóricas actuales de la educación universitaria e incorpora los aspectos pertinentes del Plan Estratégico de Desarrollo y las disposiciones legales vigentes y como metodología, utiliza la formación por competencias. El modelo educativo institucional (MEI) para la universidad es la fuerza orientadora de su ser y que hacer universitario y encuentra su fin último en la sociedad.

Palabras clave: Modelo educativo, Reforma, Universidad, Docente, Estudiante.

Abstract: The objective of this work is to study and analyze the reform process of the educational model of the National University of Agriculture. In this sense, interviews were conducted with different actors such as teachers, graduates and students so that they could provide their point of view of the educational model of the UNA, likewise reflection sessions were held with experts in the educational area to analyze the system that the UNA has. university in the educational field, the reform adhered to the tunning project for latin america, which has a structured methodology that facilitates the understanding of academic study plans and their mutual comparison. From this scenario, the National University of Agriculture (UNA), with the inputs obtained from various sources, will build the educational model that is based on current theoretical conceptions of university education and incorporates the relevant aspects of the Strategic Development Plan and current legal provisions. and as a methodology, it uses competency-based training. The institutional educational model 
(MEI) for the university is the guiding force of its being and what to do as a university and finds its ultimate goal in society.

Keywords: Educative model, Reform, Teacher, Student.

\section{INTRODUCCIÓN}

El cambiante contexto de inicios de milenio plantea grandes y variadas exigencias a las sociedades contemporáneas, particularmente a las denominadas "subdesarrolladas". La mayoría de estas naciones, antiguas colonias de los siglos XVI-XIX, se perfilan a inicios del siglo XXI arrastrando problemas sociales, económicos y políticos que encuentran sus raíces profundas en esa época. Con este pesado lastre se enfrentan al nuevo milenio en el contexto de una economía mundial englobante, competitiva y discriminadora, que basa cada vez más su desarrollo en el conocimiento, el progreso técnico, la innovación y la creatividad (Rodríguez 2006-2010)

El nuevo contexto mundial en materia educativa supone nuevos retos, especialmente para los docentes, quienes son protagonistas de la acción educativa, por lo cual sus desafíos son múltiples, complejos, diversos y propios de cada situación que les toca enfrentar (Caballero, 2001).

Actualmente, existe la necesidad creciente de que todos los docentes, ya sea los que están en ejercicio como aquellos que ingresarán al sistema superior, estén en condiciones de aprovechar los diferentes recursos tecnológicos para incorporarlos en forma efectiva en su práctica profesional. Por ello, levantar unos estándares en estas materias permitirá orientar y mejorar la forma en que los recursos tecnológicos son incorporados a la docencia.

Este documento presenta una propuesta de modelo educativo de formación para docentes de educación superior en la universidad nacional de agricultura localizada en el municipio de Catacamas departamento de Olancho, Honduras. Este modelo se constituye en una proyección del modelo de referencia: formación de docentes Conexiones dirigido a docentes de educación básica y media, hacia la educación superior.

El propósito de la reforma es establecer las medidas o estrategias para conseguir algún objetivo en materia educativa. Estas representan los esfuerzos que llevan a cabo las autoridades y los representantes ciudadanos para elevar la calidad educativa, ampliar la cobertura, mejorar la atención a ciertos grupos poblacionales o cualquier otro objetivo explícito que se persiga (Santibáñez 2008).

\section{Reformas en américa latina}

Nadie puede hoy dudar que realmente en América Latina existan los procesos de transformación y de reforma educativa a. La presencia de un proceso de transformación y de reforma al interior de las instituciones estatales encargadas de impulsarlas son, en cambio, más allá de las dos características señaladas mayor continuidad y cambio de perfil de los equipos de gobierno menos evidentes. Sin embargo, esas dos características, combinadas con la necesidad de rendir cuentas, siempre presente en las sociedades democráticas, y con el hecho de que haya "algo para hacer ( Braslavsky, C., \& Cosse, G. 1996).

El actual modelo económico, sociopolítico y cultural de la globalización responde a una política de renovada concentración del capital en las elites mundiales. El intelectual norteamericano Noam Chomsky (2001) afirma que no es posible conocer ni explicar los objetivos de los programas y las políticas públicas

Notas DE AUTOR 
de Latinoamérica, sin considerar las recomendaciones de las agencias internacionales de financiamiento, que detallan específicamente cada área de la vida de los países no desarrollados, condicionando los empréstitos al cumplimiento de sus recomendaciones. Los gobiernos de Latinoamérica se han visto obligados a aplicar políticas similares para liberar los sectores productivos estructurales, reservados con anterioridad exclusivamente al Estado y, en un segundo momento, para liberar los sectores comercial, financiero y de servicios.

La actual concepción de la calidad en la educación se fundamenta en la gestión de la calidad total, paradigma altamente exitoso en las empresas privadas. Desde este enfoque se han analizado las causas de la "ineficiencia" de las empresas públicas y se han propuesto soluciones a las mismas. La idea es que para mejorar la calidad de los servicios y productos se debe impactar a los centros públicos con la lógica del libre mercado. El sistema tiene los siguientes componentes: liderazgo, estrategia, políticas, gestión personal, recursos, procesos y procedimientos pero también incluye la satisfacción de las expectativas de los clientes y del personal (guerra 2006).

En consecuencia, el modelo educativo necesita cambios que le hagan pasar de la estandarización a la personalización, considerando las necesidades del estudiante, de tratar de introducir información en la mente del estudiante a ayudarlo a comprender y utilizar las capacidades de su inteligencia, de un aprendizaje pasivo a uno activo que, en lugar de estar dirigido por el profesor, sea controlado por el estudiante o compartido con el docente, de un aprendizaje descontextualizado a tareas auténticas, conectadas con la realidad y significativas, de considerar el aprendizaje como un proceso finito en el tiempo y el espacio a una actividad continua que se extiende a lo largo de la vida (Gutiérrez 2003).

\section{Ejes transversales del Modelo Educativo de la UNA}

El modelo educativo de la UNA destaca su acción con los siguientes ejes transversales:

A. Aprendizaje centrado en el estudiante

El proceso educativo centrado en el aprendizaje constituye una de las líneas que conforman el Modelo Educativo de la UNA. Este proceso, considera que todo ser humano tiene un gran potencial susceptible de desarrollarse cuando muestra interés por aprender; este proceso se sustenta en los cuatro pilares de la educación, que propone Delors: aprender a conocer, es decir, adquirir los instrumentos de la comprensión; aprender a hacer, para poder influir sobre el propio entorno; aprender a convivir juntos, para participar y cooperar con los otros en todas las actividades humanas; y por último, aprender a ser, un proceso fundamental que recoge elementos de los tres anteriores. Integrar los cuatro tipos de aprendizajes en el proceso educativo conlleva a la consecución de un aprendizaje significativo en los estudiantes (Delors, 1996).

La Declaración Mundial sobre la Educación Superior en el Siglo XXI: visión y acción (UNESCO 1998), plantea la importancia de centrar la educación universitaria en el aprendizaje del estudiante y no en la enseñanza tradicional. Dada la situación actual de los procesos de cambio y la nueva sociedad del conocimiento, las universidades deben comprender la necesidad de diseñar estrategias pedagógicas innovadoras, que aborden el pensamiento crítico, la creatividad y el trabajo en equipo, para propiciar vínculos de colaboración entre la universidad y la comunidad. Centrar la educación en el aprendizaje se traduce en formar ciudadanos bien informados y profundamente motivados, provistos de sentido crítico, capaces de analizar los problemas de la sociedad, de aportar soluciones, aplicar éstas y asumir responsabilidades sociales.

Finalmente tal como se expresa en la Declaración de la UNESCO (1998), la educación universitaria tiene que adaptar sus estructuras y métodos de enseñanza a las nuevas necesidades. Se trata de pasar de un paradigma centrado en la enseñanza y la transmisión de conocimientos a otro centrado en el aprendizaje y el desarrollo de competencias transferibles a contextos diferentes en el tiempo y en el espacio.

B. Ética universitaria 
La UNA asume la ética universitaria, así como el compromiso y la práctica de los valores: identidad, responsabilidad, honestidad, respeto. Asume también sus principios y el compromiso de emplear los medios y componentes necesarios para la construcción de un ambiente ético en los estudiantes universitarios, de tal manera que, y como la impronta de un ejemplo que viene de sus orientadores, forme parte de ellos en su desempeño laboral y en los diferentes ámbitos de su vida. El compromiso ético universitario debe estar presente en todas y cada una de las misiones y funciones que hoy cumple la educación superior en la UNA. Desde el enfoque de aprendizaje ético que presentamos, la intencionalidad pedagógica y ética que pretendemos incorporar a la formación universitaria no se agota en el ámbito de los medios y de los recursos de aprendizaje, ni en la identificación de los fines que creemos corresponde alcanzar a la universidad desde un punto de vista ético. Se trata de establecer relaciones entre los fines y objetivos de aprendizaje éticos y los medios y recursos disponibles para su consecución (Bara F. Estrada M. Martín M. 2002).

C. Formación basada en competencias

Según Tobón (2008) las competencias son un enfoque para la educación y no un modelo pedagógico, pues no pretenden ser una representación ideal de todo el proceso educativo, determinando cómo debe ser el tipo de persona a formar, el proceso instructivo, el proceso desarrollador, la concepción curricular, la concepción didáctica, la concepción epistemológica y el tipo de estrategias didácticas a implementar. $\mathrm{Al}$ contrario, las competencias son un enfoque porque sólo se focalizan en unos determinados aspectos conceptuales y metodológicos de la educación y la gestión del talento humano

Para el modelo educativo de la Universidad Nacional de Agricultura, se entenderá por competencias el "conjunto de conocimientos, habilidades y actitudes articulados requeridos para llevar a cabo una tarea determinada, el desempeño de una función específica o ejercer una profesión" de manera efectiva y éticamente responsable, con creatividad e innovación.

D. Investigación

La investigación científica es un proceso que, mediante la aplicación del método

científico, procura obtener información relevante y fidedigna, para entender, verificar, corregir o aplicar el conocimiento (Tamayo 2004)

La investigación constituye una función esencial y obligatoria de la UNA. La fomenta y realiza, respondiendo a través de la investigación científica, tecnológica, humanística con enfoques cuantitativos y cualitativos en líneas de investigación pertinentes a la Realidad social, necesidades del país, solución de problemas, satisfacción de demandas, desarrollo humano, y desarrollo sostenible. La formación de profesionales de excelencia requiere la investigación y la docencia como ámbitos de desarrollo permanente de la actividad académica de los profesores.

E. Innovación universitaria

Los procesos de innovación respecto a la utilización de las tecnologías de información y comunicación (TIC) en la docencia universitaria suelen partir, la mayoría de las veces, de las disponibilidades y soluciones tecnológicas existentes. Sin embargo, una equilibrada visión del fenómeno debería llevarnos a la integración de las innovaciones tecnológicas en el contexto de la tradición de nuestras instituciones; instituciones que, no olvidemos, tienen una importante función educativa (Ibáñez, 2004)

La UNA se plantea, una parte, la necesidad de establecer nuevas formas y enfoques para generar y facilitar la innovación y, por otra, el de poner a disposición de los docentes y estudiantes recursos e instrumentos adecuados para implementar dicha innovación.

F. Cultura de calidad educativa

$\mathrm{Al}$ respecto, la UNA toma en cuenta que "la calidad es la adecuación del ser y del quehacer de la educación superior, a su debe ser" (UNESCO, 1998), donde lo esencial es definir qué debe ser y hacer una universidad dentro del contexto en que se desarrolla. La educación es un "sistema complejo", es decir, un sistema en el cual, en la totalidad o la unidad, existe la diversidad, por lo que la unidad o totalidad es la síntesis de múltiples 
determinaciones. Un sistema complejo se caracteriza porque contiene múltiples subsistemas fuertemente conectados (Aguerrondo 1993).

\section{ARTICULACIÓN DEL MODELO EDUCATIVO Y LA LEGISLACIÓN}

La articulación del Modelo Educativo de la UNA y la legislación educativa del país es importante, ya que la educación es donde el pueblo, con conciencia política, se incorpora activamente en los procesos de cambio y transformación social, donde priva la supremacía del hombre por encima del mercado y del capital. Una nueva sociedad en la que el ser humano, como centro de la misma, deja de ser la mercancía predilecta del capitalismo y pasa a ser sujeto de su propia transformación.

A. Identidad y cultura institucional

La identidad de la UNA lo define la misión y visión institucional, se describen a continuación y por los procesos de reforma de la universidad, estas se revisarán y actualizarán para responder a servicios de calidad institucional.

B. Normativa y documentos de gestión

El Modelo Educativo de la UNA se fundamenta en las leyes siguientes: La Constitución de la República

Las Normas Académicas, Estatuto Académico y sus Reglamentos La Ley de Educación Superior y su Reglamento

Las Normas Académicas del Nivel de Educación Superior.

La Constitución de la República, Título III de las Declaraciones, Derechos y Garantías, Capítulo VIII, de la Educación y Cultura:

Art. 151. La educación es función esencial del Estado para la conservación, el fomento y difusión de la cultura, la cual deberá proyectar sus beneficios a la sociedad sin discriminación de ninguna naturaleza. La educación nacional será laica y se fundamentará en los principios esenciales de la democracia, inculcará y fomentará en los educandos profundos sentimientos hondureñistas y deberá vincularse directamente con el proceso de desarrollo económico y social del país.

Art. 162. Por su carácter informativo y formativo, la docencia tiene una función social y humana que determina para el educador responsabilidades científicas y morales frente a sus discípulos, a la institución en que labore y a la sociedad.

Las Normas Académicas, Estatuto Académico y sus Reglamentos

Por los procesos de reformas en la UNA, estas normativas fueron presentadas al Consejo de Educación Superior, para reformarlas según acuerdos de Ingresos: Normas Académicas 1600-191-2006 de fecha 24/04/2006, Estatuto Académico No.2660-270- 2013 de fecha 20/03/2013.

\section{Ley de Educación Superior}

\section{Capítulo I - Naturaleza y Fines}

Art. 3. "La educación superior tiene como fines la investigación científica, humanística y tecnológica; la difusión general de la cultura; el estudio de los problemas nacionales; la creación y transmisión de la ciencia y el fortalecimiento de la identidad nacional".

Capítulo II - Principios de la Educación Superior

Art. 5. "La docencia, la investigación y la extensión son elementos esenciales y concurrentes en el proceso educativo del nivel superior."

Art. 6. "La educación superior tiene como contenido característico, el dominio de sus disciplinas, el incremento del saber y la conservación, creación y transformación de la ciencia, la filosofía, las artes, las 
técnicas y demás manifestaciones de la cultura y la capacidad de proyección en beneficio de la sociedad, en cuya transformación debe participar."

\section{Normas Académicas del Nivel de Educación Superior}

En el capítulo II referente a la Naturaleza y los Objetivos, el artículo 2 declara que: "Las Normas Académicas de la Educación Superior constituyen el instrumento que regula el desarrollo de la actividad académica de este nivel de la educación nacional, bajo una concepción dinámica de los procesos formativos".

El artículo 4 en relación a los Objetivos de estas Normas expresa que: "orientarán las actividades académicas del nivel de educación superior por medio de la integración funcional de la docencia, la investigación y la extensión".

El capítulo IV hace referencia a las funciones básicas del nivel: la docencia, investigación y extensión, y conceptualiza cada uno de los elementos que se conjugan en el desarrollo curricular de las diferentes disciplinas, siendo estos los objetivos educacionales, las metodologías, técnicas y recursos de enseñanza, las formas de evaluación, la modalidad presencial y a distancia.

C. Concepción del proceso enseñanza-aprendizaje

Este proceso para la UNA se entiende como la interacción comunicativa, intencionada y planificada para la promoción de cambios en los sujetos, de acuerdo a la propuesta formativa que define el currículum en los dominios teóricos, procedimentales y actitudinales.

Los programas de formación presentan una selección intencionada de:

- Una sólida base disciplinaria y metodológica proveniente de la investigación, los avances de la disciplina y los requerimientos del contexto profesional.

- Experiencias pedagógicas conducentes a desarrollar saberes procedimentales propios de la disciplina y del ejercicio profesional.

- Saberes actitudinales referidos principalmente a los valores éticos frente al aprendizaje y al ejercicio de la profesión.

Entre los componentes primordiales y asociados al proceso enseñanza-aprendizaje están las estrategias, recursos y dispositivos didácticos y evaluativos: Las estrategias y métodos pedagógicos: que comprenden un conjunto de acciones y procedimientos que conducen y facilitan el logro de resultados de aprendizaje. Los métodos pedagógicos usados en el contexto de este Modelo Educativo de la UNA fomentan el aprendizaje activo de los estudiantes, a través de experiencias de aprendizaje mediadas que posibilitan la construcción de conocimientos para su trasformación o aplicación, así como la práctica de la investigación como generadora de nuevo conocimiento.

Los recursos y dispositivos didácticos, comprenden los materiales y medios usados intencionalmente para facilitar los procesos de enseñanza-aprendizaje. Estos proveen el acceso a la información para la construcción de conocimientos y desarrollo de habilidades. Entre estos el Modelo asume la necesidad de una incorporación activa de las Tecnologías de la información y comunicación (Tics) en los procesos formativos de la UNA.

\section{Conclusiones}

1. El modelo educativo de la UNA deberá estar integrado incorporando las

conceptualizaciones de los modelos centrados en los aprendizajes, de su enfoque por competencias y aprendizaje significativo.

2. La UNA en su proceso de cambios y transformación, su MEI contribuirá a formar un ser

social, que con nuevas aptitudes profesionales, enfoque su sentido de pertenencia, descubra la finalidad de su ser, saber, y hacer, determine sus necesidades de seguir aprendiendo, reconozca el estado del medio social, 
empresarial en el que interviene y se desarrolla, como forma de entender la realidad que le rodea, reflexionar críticamente sobre ella y decidir con autonomía e intelecto.

3. La elaboración de los instrumentos constituyó un ejercicio clave para obtener

información sistematizada de acuerdo a las dimensiones del modelo educativo y pedagógico. Desde el enfoque democrático de la evaluación la participación de los actores en esta etapa fue valiosa, pero se considera que podría haber sido más amplia, por lo que se concluye que la participación de los actores debe de ser continúa durante el proceso y no solo en determinadas fases.

4. El nuevo modelo educativo de la UNA brindará la oportunidad para que la población de

la región identifique y experimente mejores niveles de desarrollo económico y cultural.

\section{REFERENCIAS BIBLIOGRÁFICAS}

Aguerrondo, I. (1993). La calidad de la educación: ejes para su definición y evaluación. Revista interamericana de desarrollo educativo, 37(116), 561-578.

Bara, F. E., Estrada, M. R. B., \& Martín, M. M. (2002). La universidad como espacio de aprendizaje ético. Revista Iberoamericana de educación, (29), 17-44.

Braslavsky, C., \& Cosse, G. (1996). Las actuales reformas educativas en América Latina: cuatro actores, tres lógicas y ocho tensiones (No. 5). Santiago de Chile: Preal.

Caballero, S. (2001). Retos de la maestra y maestro del siglo XXI. Revista Educación, 1(24), 47-52.

Chomsky, N. (2001, 25 de enero). La globalización en América Latina. Trabajo presentado en la Cátedra Latinoamericana Julio Cortázar, Guadalajara, Jal., México.

Delor, J. (1996). Informe de la UNESCO de la comisión internacional sobre la educación en el siglo XXI, presidida por Jacques Delor.

Guerra, S. L., \& Chávez, M. F. (2006). Las reformas educativas neoliberales en Latinoamérica. Revista electrónica de investigación educativa, $8(1)$.

Gutiérrez, O. (2003). Enfoques y modelos educativos centrados en el aprendizaje. El proceso educativo desde los enfoques centrados en el aprendizaje, 3.

Ibáñez, J. S. (2004). Innovación docente y uso de las TIC en la enseñanza universitaria. RUSC. Universities and Knowledge Society Journal, 1(1), 3.

J, S. (1998). La virtualización de la Universidad: ¿Cómo podemos transformar la educación superior con la tecnología? Caracas: IESALC-UNESCO.

Rodríguez, R. H., Solís, M. A., \& Moncada, G. Revisión teórica sobre lA Investigación Acción y sus desafíos PARA lA Formación docente en Honduras. Investigación educativa en la UPNFM: 2006-2010, 172.

Santibáñez, L. (2008). Reforma educativa: el papel del SNTE. Revista mexicana de investigación educativa, 13(37), 419-443.

Tamayo, M. (2004). El proceso de la investigación científica. Editorial Limusa.

Tobón, S. (2008). La formación basada en competencias en la educación superior: el enfoque complejo. México: Universidad Autónoma de Guadalajara. 